Review

\title{
Application of Solution NMR Spectroscopy to Study Protein Dynamics
}

\section{Christoph Göbl ${ }^{1}$ and Nico Tjandra ${ }^{2, *}$}

1 Institute of Chemistry, Organic and Bioorganic Chemistry, University of Graz, Heinrichstrasse 28, A-8010 Graz, Austria; E-Mail: christoph.goebl@uni-graz.at

2 Laboratory of Molecular Biophysics, National Heart, Lung and Blood Institute, National Institutes of Health, 50 South Drive, Bethesda, MD 20892, USA

* Author to whom correspondence should be addressed; E-Mail: tjandran@nhlbi.nih.gov; Tel.: +1-301-402-3029; Fax: +1-301-402-3405.

Received: 30 January 2012; in revised form: 13 March 2012 / Accepted: 16 March 2012 / Published: 22 March 2012

\begin{abstract}
Recent advances in spectroscopic methods allow the identification of minute fluctuations in a protein structure. These dynamic properties have been identified as keys to some biological processes. The consequences of this structural flexibility can be far-reaching and they add a new dimension to the structure-function relationship of biomolecules. Nuclear Magnetic Resonance (NMR) spectroscopy allows the study of structure as well as dynamics of biomolecules in a very broad range of timescales at atomic level. A number of new NMR methods have been developed recently to allow the measurements of time scales and spatial fluctuations, which in turn provide the thermodynamics associated with the biological processes. Since NMR parameters reflect ensemble measurements, structural ensemble approaches in analyzing NMR data have also been developed. These new methods in some instances can even highlight previously hidden conformational features of the biomolecules. In this review we describe several solution NMR methods to study protein dynamics and discuss their impact on important biological processes.
\end{abstract}

Keywords: protein; protein structure; protein dynamics; protein interaction; solution NMR spectroscopy 


\section{Introduction}

The rapid advancement of technology in the biomedical research field permits the study of biological processes in much more detail than was previously thought possible. In almost all cases the availability of structural information at the molecular or atomic level is essential to provide a detailed understanding of molecular mechanisms governing those processes. The chemical and physical characters of proteins or nucleic acids, as well as their activity and behavior are ultimately attributed to their structures. However, in the last few years it has become more evident that dynamic properties of these biological molecules play as important of a role as their structures. In addition to the spatial arrangement, dynamic fluctuations have been identified as a driving force for many types of molecular interactions. Most biological processes in a cell are tightly regulated, such as signaling, transcription regulation or immune response and dynamic contributions have been found as a major regulatory control.

One example is the interaction between kinase $\mathrm{p} 110 \alpha$ and its activator $\mathrm{p} 85 \alpha$, which involves the loop regions of the molecules. Mutations in this interaction site have been shown to ultimately lead to cancer [1]. Detailed studies have indicated that no major structural changes were observed with these mutants, but rather altered dynamic behavior causes modified kinase activity [2]. Therefore, structural data alone does not reflect on the different behavior of the mutants, rather the analysis of protein dynamics leads to insights into the exquisite regulatory mechanisms.

New methodological approaches focus more on determining structural fluctuations and their consequences to the function of proteins, than just the three-dimensional static structures. According to the energy landscape model, a protein ensemble displays different populations of conformational coordinates (Figure 1). The population of the ensemble follows the Maxwell-Boltzmann distribution, and the energy barrier between different sub-conformations determines their rate of interconversion. A key hypothesis of recent investigations is that only a certain state, characterized by its temporary spatial arrangement, contributes to a specific function. This leads to a complex, concerted picture of protein motions featuring different timescales and amplitudes.

A popular model of enzyme activity, interpreted in the context of the energy landscape model, is a protein as a shapeable scaffold that is forced to change its structural arrangement during successful interactions to a binding partner [3]. This "induced fit" model is proposed since the out-dated "key-lock" model fails to explain enzymatic reactions with slightly modified substrates. These different models can further be evaluated due to recent advances that allow direct measurements of protein ensembles and reveal the dynamic nature of molecules. More recent results suggest that a protein ensemble intrinsically populates several conformations and therefore conformational selection seems to be the driving force for protein interactions [4]. Some striking examples will be discussed.

Characterization of even minute fluctuations about static structures has been made possible through major advances in technologies. X-ray crystallography, with the advent of high energy sources, is almost routinely used for structure determination of well-folded biomolecules. For studying dynamic processes, crystallization of two endpoints of a reaction, such as the unbound and bound state of an enzyme, allow a linear interpretation of rearrangements that take place during binding processes. Picosecond time resolved X-ray crystallography [5] and time resolved wide-angle X-ray scattering [6] were applied to study conformational changes in $\mathrm{CO}$ saturated and deoxygenated myoglobin. 
Similarly, improvement in cryo-electron microscopy has resulted in better resolution images to allow reconstruction of three-dimensional models at higher resolution than was previously possible. In addition, nuclear magnetic resonance (NMR) aided by improvements in protein isotopic labeling methodologies has been able to study the structure and dynamics of large proteins and protein complexes even at the size of a proteasome [7] as well as proteins at residue specific levels in living cells $[8,9]$.

Figure 1. Schematic free-energy landscape of proteins. Dynamic models of proteins are visualized in a free-energy landscape as a function of conformational coordinates. This simplification involves a certain set of sample conditions. Minima correspond to well-defined, stabilized states whereas maxima reflect short-lived transition states. The height of the energy barriers is proportional to the timescale that is necessary to stochastically convert between states. Any influence such as molecular interaction leads to redistribution within the energy landscape and/or modification of the profile. The volume of the red spheres illustrates a hypothetical population of conformers.

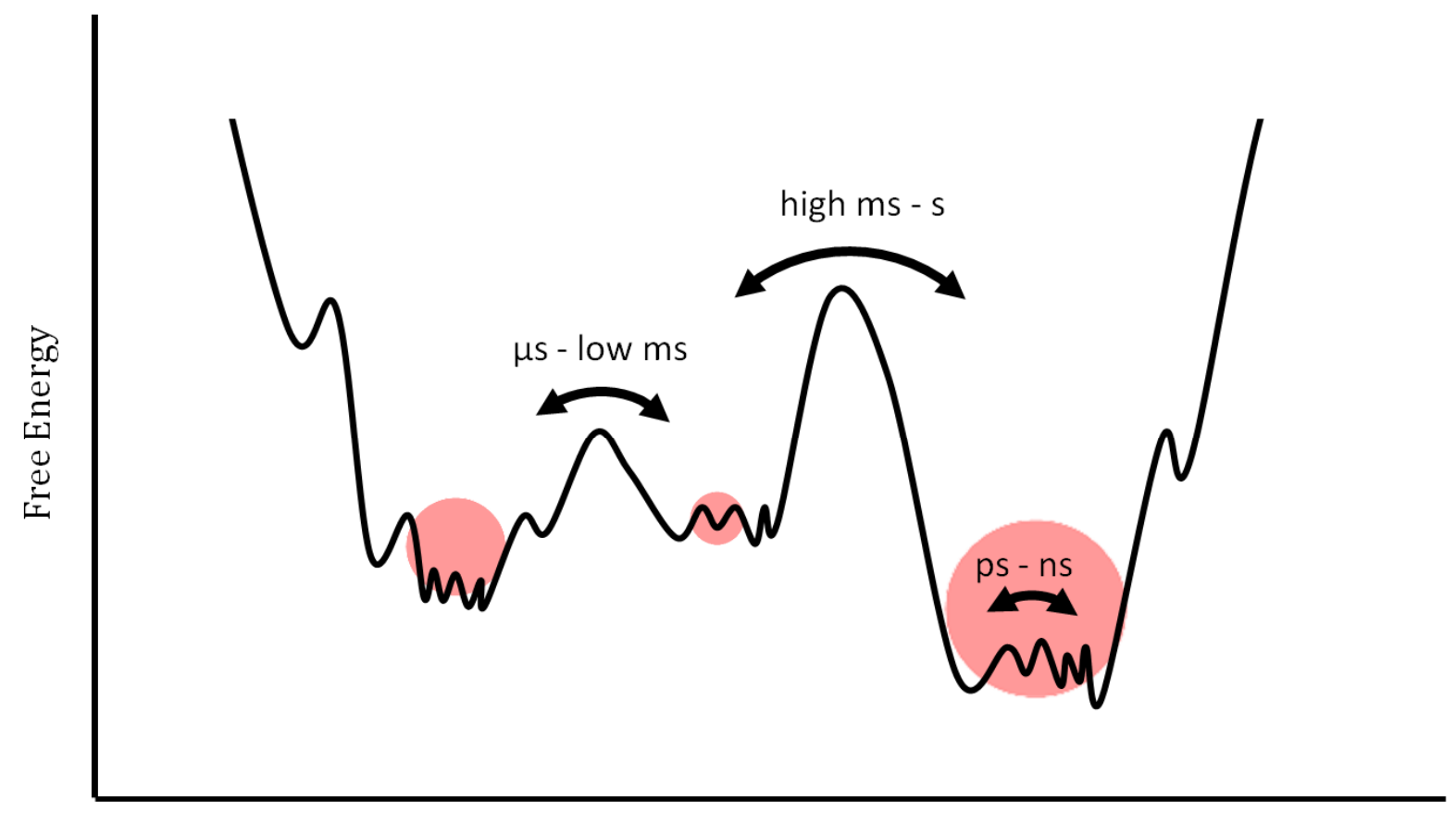

Conformational coordinate

Beside these high-resolution methods, several approaches were developed to quantify global spatial changes. Small angle X-ray scattering (SAXS) applications allow the determination of effective shapes, that is sensitive to large dynamic structural changes in proteins [10,11]. Incorporation of fluorescence probes allows Förster Resonance Energy Transfer (FRET) measurements even on a single molecule level and determination of conformational changes through the associated distance fluctuations [12]. Similar to FRET, although arguably not as sensitive, Electron Paramagnetic Resonance (EPR) measurements allow determination of intra- and intermolecular distances and are widely used when studying metal containing proteins [13] or after the introduction of stable radicals [14]. 
We will concentrate on NMR dynamics measurements that correlate thermodynamic properties of a protein to its function. The measurement of dynamic parameters can be divided into slow, medium and fast motions, depending on their relationship to the NMR timescale. Slowly exchanging equilibria can be detected in a straightforward manner if the NMR chemical shift and therefore the structural conformations are different during detection of the signal. Additionally, measuring successive experiments and fitting a change of the signal to the parameter of interest can also detect slowly changing observables. If dynamic parameters change within the duration of the NMR signal acquisition, that is on the order of a hundred milliseconds, it will influence how the NMR signal will behave in response to a certain perturbation. A careful measurement of this response leads to the detection of medium timescale motions in the range of $\mu$ s to ms. Fast motions (faster than ns) can only be deduced from NMR relaxation measurements. NMR relaxation times depend on the fluctuations of the nuclear interactions (such as nuclear dipoles or chemical shift anisotropies) with respect to the static magnetic field due to the overall rotational diffusion of the protein in solution as well as local fast motions in the protein.

\section{Slow Dynamic Interchange between Various Conformations}

Processes that occur at timescales much slower than the NMR acquisition time will permit the measurements of various states independently. In addition, the measurements from successive experiments that track changes within this slow timescale allow the determination of kinetic parameters.

Hydrogen to deuterium exchange experiments have long been recognized to report on the dynamic behavior of a protein backbone [15]. The basic principle is the exchange of the labile protein backbone amide group between proton and deuterium from the solvent. This is achieved by the rapid transfer of the protonated protein into $\mathrm{D}_{2} \mathrm{O}$ buffer and followed by successive measurements of ${ }^{1} \mathrm{H}-{ }^{15} \mathrm{~N}-\mathrm{HSQC}$ two-dimensional NMR spectra. Intensity changes over time can be translated into a hydrogen exchange rate that yields information about the solvent accessibility and local structure stability (i.e., hydrogen bonding) that can be influenced by dynamic processes. This type of experiment is also often used to study folding events [16,17] where NMR spectroscopy is combined with hydrogen exchange pulse labeling, a stopped-flow mixing method to prepare samples with very short exchange time [18]. This approach has further led to the development of fast NMR data acquisition methods, allowing to record two-dimensional spectra in seconds and the detection of faster exchange rates with higher accuracy [19].

Different conformers that exchange slower than about one hundred milliseconds, typical of NMR data acquisition, will allow the observation of separate NMR signals associated with each conformer (Figure 2). Proline cis-trans isomerization occurs within hundreds of milliseconds because of the high-energy barrier between the two possible states. It is typical to find 5\% of one population relative to the other in protein structures. This equilibrium can be shifted completely towards the low populated conformation in some cases. With proper regulation, proline cis-trans isomerizations can act as molecular switches that lead to conversion between two states of different functional relevance [20]. 
Figure 2. Two resonances are observed at very slow exchange. Panel A displays the hypothetical free induction decay (FID) of two different resonances for a residue that interchanges slower than the signal is being detected. A superposition of the time resolved signal is observed (panel B) and corresponds to two peaks in the Fourier transformed frequency spectrum (panel C). In favorable cases, intense peaks within the subspecies are found and even allow structure determination of each conformer (panel D).

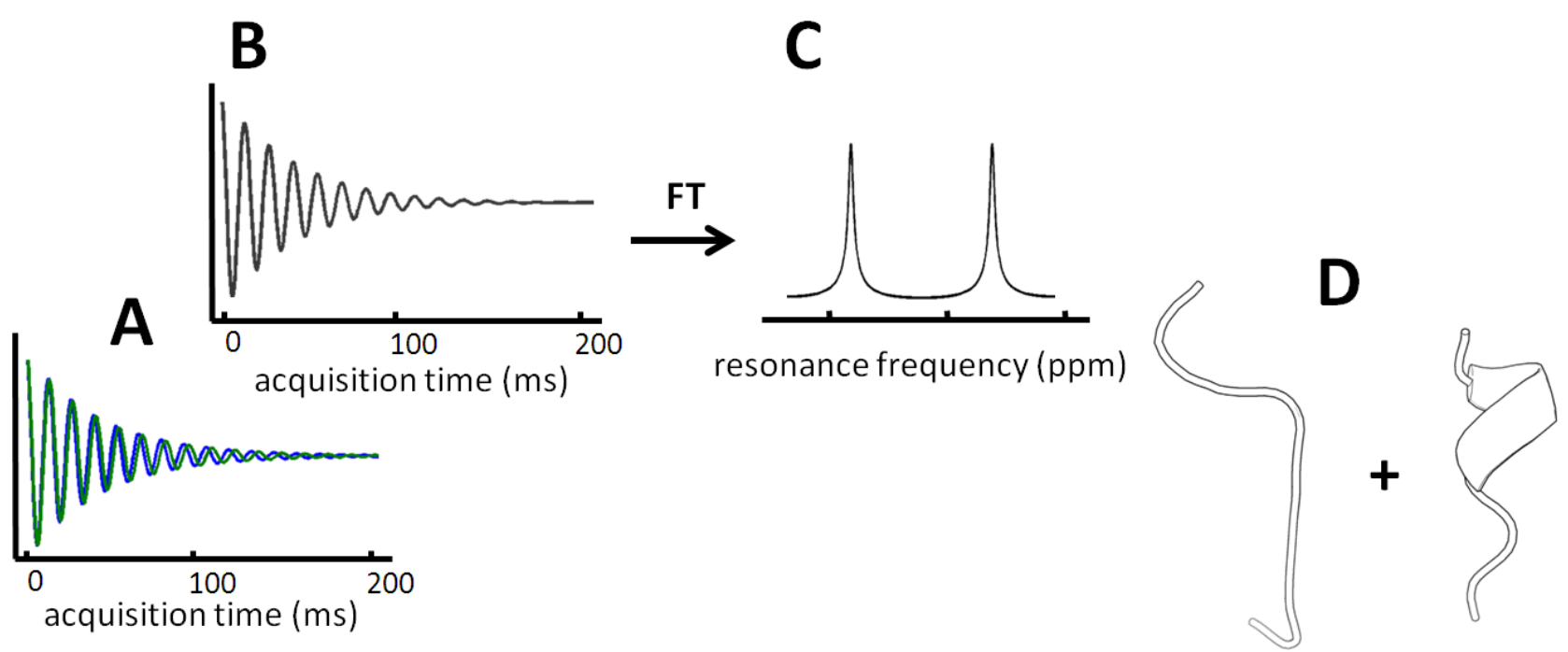

Lummis et al. highlighted the regulatory function of a proline residue within a transmembrane protein [21]. Binding of different neurotransmitter to 5-hydroxytryptamine type 3 (5-HT3) protein receptors switches an ion channel between its open and closed state. The protein consists of two domains, the ligand binding domain pointing to the cytoplasm that allows substrate interaction and a transmembrane domain that shuttles the desired ion. It is a single proline residue in a loop connecting helix 2 and 3 in the transmembrane channel that constrains active or inactive conformations (Figure 3 ). Mutation of the proline to other amino acids resulted in non-functional channels. When mutated into unnatural amino acids that display cis-trans conversion through similar ring structures, a clear correlation was found between the activity of the receptor and the cis-trans isomerization energy of these proline homologues. NMR data of helix 2 and 3 in a membrane mimicking system displayed two different sets of NMR resonances for the same proline residue. This major structural feature affects the surrounding residues that also show a duplication of NMR resonances, indicating the existence of dual conformations. The study revealed that the necessary structural rearrangement for ion transport is regulated by a proline residue that can intrinsically create stable populations of the two conformations.

While NMR can directly observe slowly exchanging conformations described above, processes that occur faster than this time scale will result in an averaging of the NMR signals from different conformers and lead to a single observed NMR resonance. Different methods are necessary to characterize these faster dynamic fluctuations. 
Figure 3. Proposed mechanism of the 5-HT3 receptor gating. The graph depicts the proposed cis-trans proline regulatory mechanism of 5-HT3. As shown on top, Helix M2 and M3 are connected via a flexible loop comprising a proline residue. Mutation of the proline residue to other amino acids inactivates the ion transport. Only amino acids that significantly allow the population of the cis-form of the backbone yielded functional channels that were detected by voltage-clamp experiments. In the bottom, a hypothetical structure of the closed and opened transmembrane channel is shown (figure reproduced with permission of reference [21]).
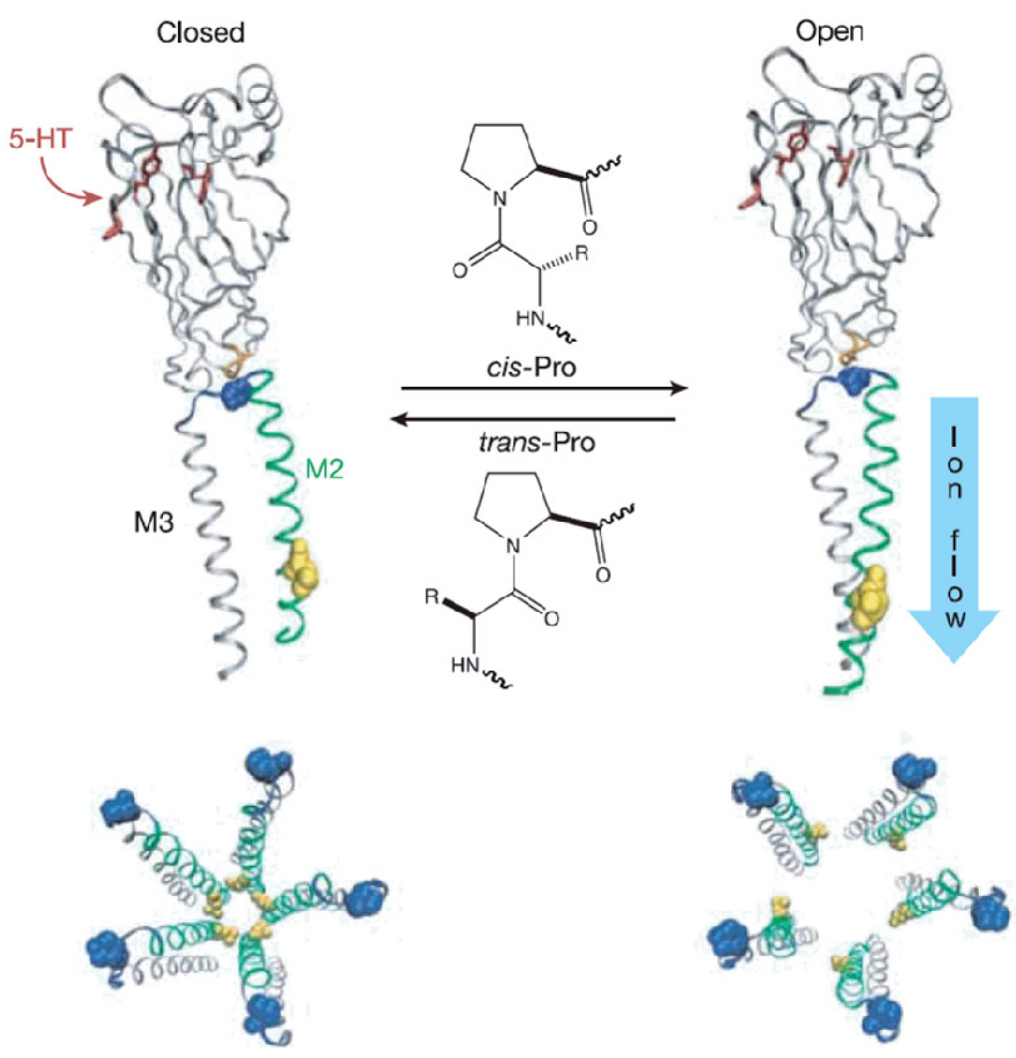

\section{Dynamic Equilibrium in Micro- to Milli-Seconds}

Dynamic fluctuations that occur at the same time scale as the NMR acquisition time will affect the evolution of the NMR signals. This is due to the interchange between the various conformers which may have different dynamic characters. There is a method to control the influence of this inter-conversion to modulate the NMR signal (the Carr Purcell Meiboom Gill, CPMG sequence [22]). This is accomplished by inverting the NMR spin state of the observed nuclei at various rates relative to the rate of the dynamic process. The amount of modulation depends on the relative population of the conformers, the separation of their resonance frequencies, and the inter-conversion rate. Measuring the NMR signal modulation accurately can allow the determination of those parameters [23]. The relative populations and exchange rates describe the kinetics of the dynamic process, while the associated resonance frequencies (chemical shifts) of the conformers provide structural information. Using this approach, it is possible to characterize a minor (thus, invisible) protein conformer that is exchanging with a major, visible conformer (Figure 4). 
Figure 4. Slow exchange of a two spin system. In line I the graph describes the principle of CPMG measurements. After excitation, chemical shift evolution leads to precession of nuclear spins in the $\mathrm{x}-\mathrm{y}$ plane. $180^{\circ}$ pulses are applied to flip the spin state and their direction of time evolution. Since the delay $t$ is constant, the signal will evolve back to the same coherence state. Relaxation rates can be determined by measurements of the signal at varying repetition numbers of the transverse block $n$ because intrinsic relaxation cannot be refocused. If there is no chemical exchange present the signal is solely built up by a single species. In line II the effect of chemical exchange is depicted. In the presence of a second state, the atom of interest is transformed into an environment of a different conformation where the chemical shift $\Omega$ differs. Because this rearrangement happens stochastically, jumping between two states in the timescale of the evolution delay leads to a modified signal without returning to the initial coherence. When measuring a series of relaxation rates by varying the chemical shift evolution period $t$, relaxation dispersion profiles are determined (III). These can be fit to gain various parameters, such as chemical shift of the second conformation, its population and exchange rate (IV).
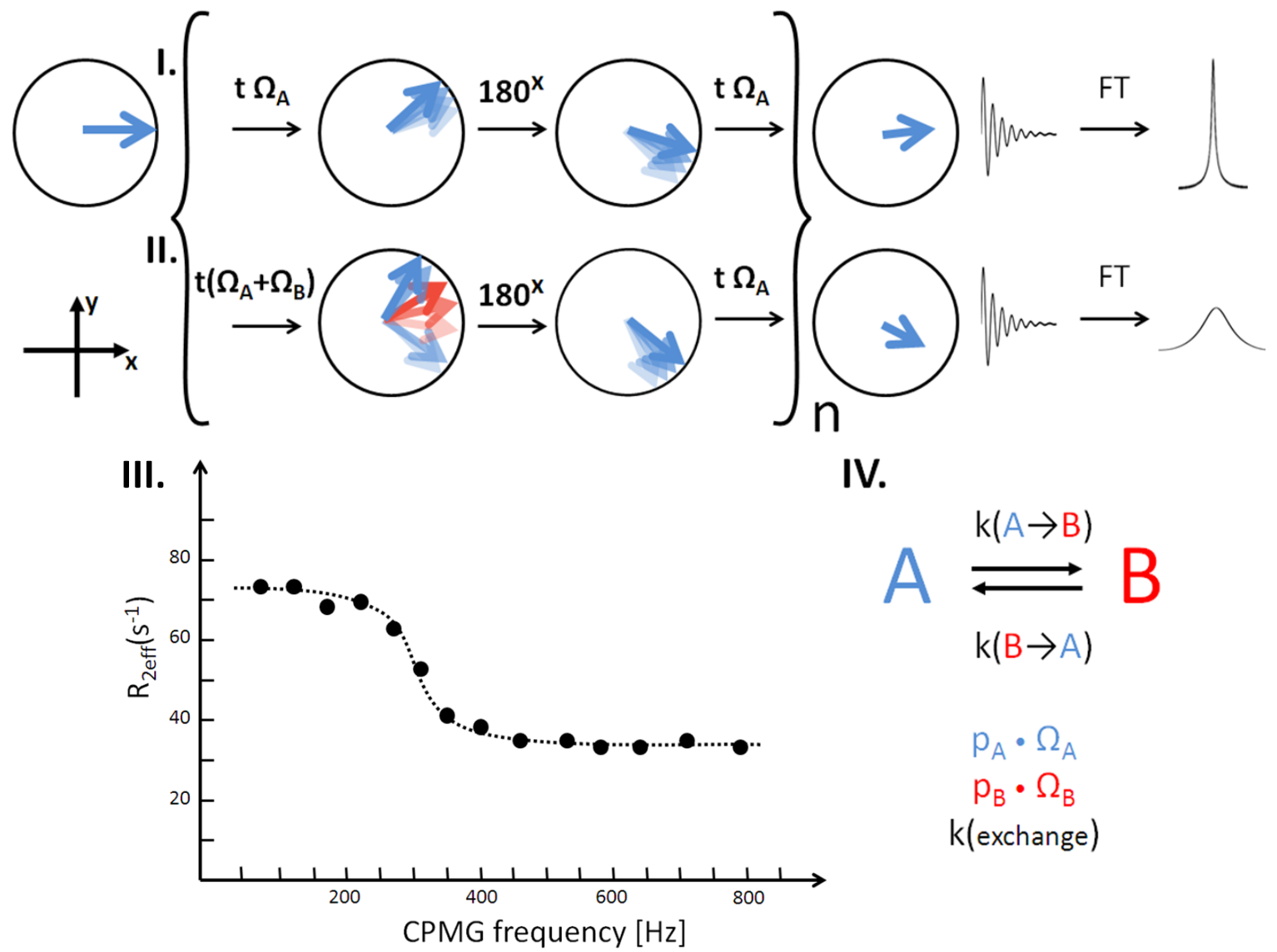

IV.

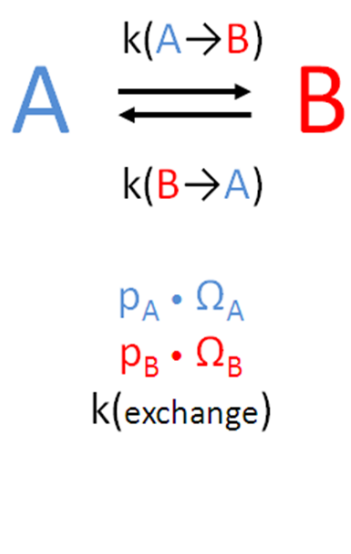

Kern and coworkers found a population shift mechanism for the nitrogen regulatory protein $\mathrm{C}$ (NtrC), a protein essential for regulating gene expression, which is activated via phosphorylation of an aspartate residue. The unphosphorylated protein was found to interconvert between active and inactive states in the low milliseconds time scale motional regime [24]. Interestingly, the aspartic acid residue that becomes phosphorylated is buried in the inactive form and hence is not accessible for modification. The dynamic rearrangement takes place predominantly in helix $\alpha 4$ which tilts, slightly 
rotates, experiences additional helical stabilization on one end and a destabilization on the other. This leads to structural reorientation and surface exposure of the active aspartate residue. Using the CPMG methods and the chemical shifts of this helix as a starting point, the chemical shifts of the opposing conformations could be extrapolated. Mutations in the active helix shifted the equilibrium between the active or inactive conformation and the chemical shift changes of these modifications correlated linearly with the enzymes transcriptional activity.

In a more recent study, an atomic resolution trajectory of this slow interchange could be experimentally verified following a computational prediction $[25,26]$. Theoretical analysis of the inter-conversion between the two states suggested transient hydrogen bonds to stabilize the intermediate conformation (Figure 5). Experimental CPMG data on a serine residue within the dynamic helix showed an inter-conversion rate of $14,000 \mathrm{~s}^{-1}$. Its protonated side chain was proposed to form a transient hydrogen bond to the neighboring aspartate during the transition. When mutating this serine to an aspartate or a glycine, the inter-conversion rate slowed down to 3,000 s $\mathrm{s}^{-1}$ which suggests a less favorable transition and a higher energy barrier between the two states. The relation to transient hydrogen bonds could also be demonstrated experimentally for other residues.

Figure 5. Calculated trajectory of helix reformation. The figure displays the predicted pathway of the structural rearrangement of nitrogen regulatory protein $\mathrm{C}(\mathrm{NtrC})$. The helix shows interconversion between active (A) and inactive states (B). Q96 is backbone hydrogen bonded in the active form, shows a transient side chain hydrogen bond during transition and is part of a loop in the inactive form. In contrast, S85 is showing a backbone hydrogen bond in the inactive state, a transient side chain hydrogen bond to D86 during transition and is part of the loop region after the helix in the active form. Several residues show millisecond dynamics during experimental CPMG measurements. Mutations of key interaction residues slow down this transition and lead to a higher energy barrier for the interconversion process. The obtained thermodynamic parameters allowed a quantitative description of the energy barrier that connects both states (figure reproduced with permission of reference [26]).

A

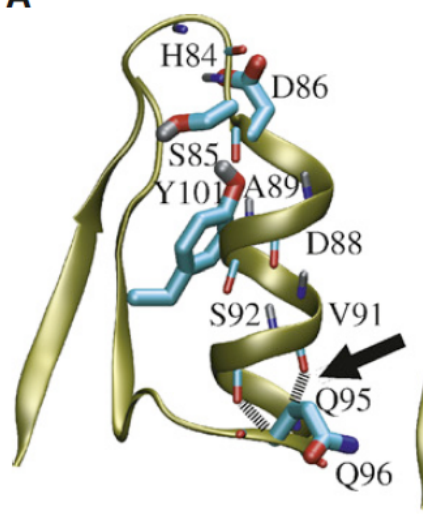

0 ps (Active)

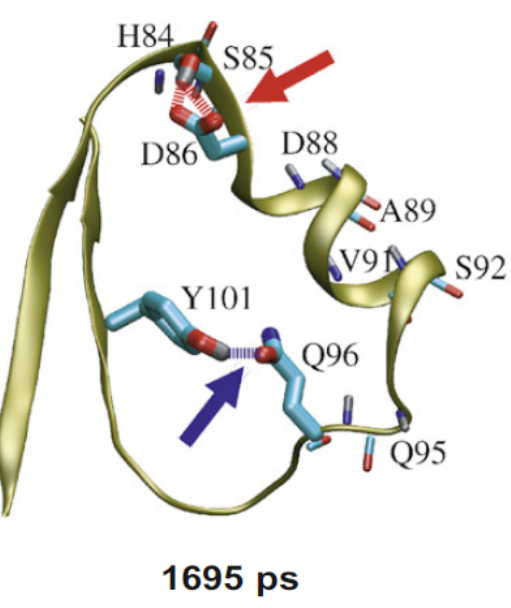

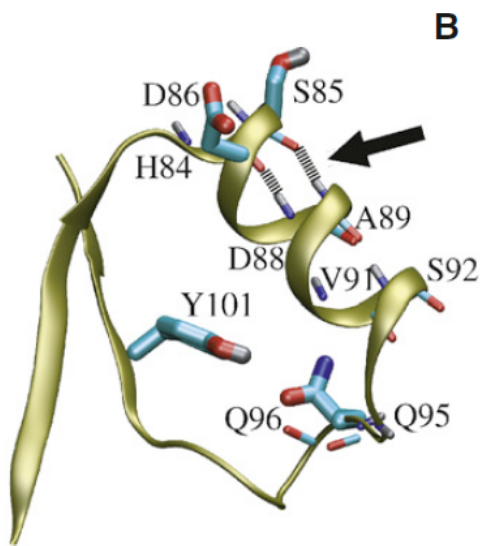

3500 ps (Inactive)

There have been a number of NMR studies that show the importance of protein dynamics in microto milli-second timescales for their biological functions [27-31]. Faster fluctuations, however, can also 
play roles in determining the thermodynamics of the biological processes. These fast fluctuations will not permit an experiment to incorporate the dynamic processes directly into a modulation of the NMR signal. The dynamic averaging occurs much faster than the NMR time scale and a different approach has to be used to extract fast motional parameters.

\section{Fast Fluctuation Compared to the NMR Time Scale}

These dynamic fluctuations are stochastic and will govern the correlation function of the magnetic dipoles. Therefore, they will influence how these magnetic dipoles relax to equilibrium after they are excited in an NMR experiment (Figure 6). A careful measurement of NMR relaxation times can reveal the time scale and amplitude of the fast local motion typically in the pico- to nano-seconds, in addition to the knowledge of the overall correlation or tumbling time of the protein [32,33]. The amplitudes of these fast local motions have been used to describe the thermodynamics associated with the function of a protein. Specifically, changes in the amplitude of the motion are related to changes in entropy [34].

Figure 6. Probing fast dynamics using relaxation effects. Panel A demonstrates overall and local reorientation of a protein in solution. The magnetic field influence of a covalently bound spin pair is highlighted. Each nucleus experiences a local fluctuating magnetic field which is governed by both types of motion. These fields are the source of relaxation (panel B). Accurate measurement of relaxation rates allows its dissection into global and local motion contributions. Through the seminal work of Lipari \& Szabo [32,33], dynamic parameters can be extracted without prior restriction to a model. The derived order parameter $S^{2}$ adopts values between unordered (0) and rigid (1). In the simplest case, the information obtained corresponds to diffusion of a bond vector in a cone with a characteristic semi-angle (panel C).

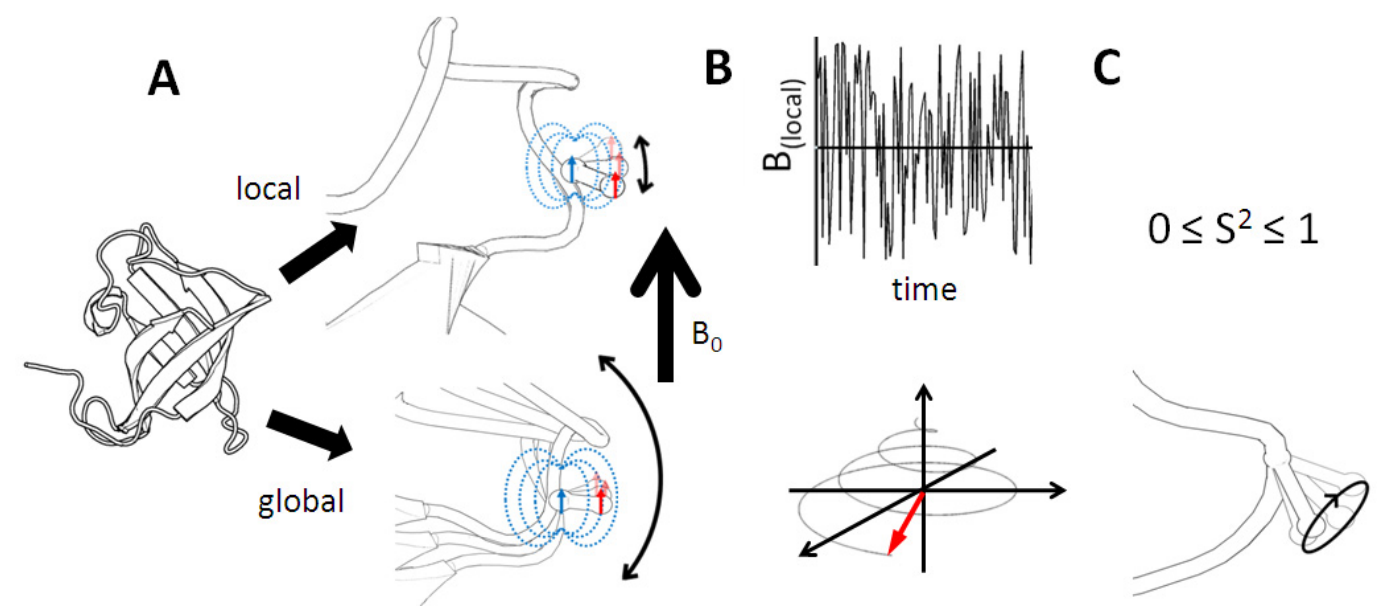

Wand and coworkers have developed an empirical calibration of the order parameter in terms of conformational entropy in the binding processes [35]. They have shown this relationship in the context of calmodulin interaction with its various targets. Calmodulin is a calcium binding protein that plays important roles in cell signaling. Calmodulin binds to a multiplicity of alpha helical proteins in a conserved mechanism by wrapping around the N-terminus of the interaction partner. Although similar Gibbs free energy $(\Delta G)$ values for calmodulin binding events are found, large variation within the 
enthalpic and entropic contributions could be observed. Picosecond to nanosecond methyl group side chain dynamics were detected in calmodulin that significantly alter after the formation of its complex with several interaction partners [36]. The total entropy changes upon binding were specified as the sum of entropy changes of calmodulin, its interaction partners, overall solvent and combined rotational and translational contributions. The latter were assumed to be constant within the different complexes because of the similar size, shape and type of interaction. By comparing the measured total entropy changes via isothermal titration calorimetric analysis versus the measured conformational entropies, a linear correlation could be observed (Figure 7) [35]. Denoted as the creation of an 'entropy meter', residue specific conformational entropy contributions to binding events could be determined experimentally. However, even though this approach provides site-specific entropy measurements, they only report on a subset of the interacting chemical groups. For instance, the NMR relaxation measurement is not sensitive to fluctuations of the methyl groups along their axis, therefore this contribution is not accounted for. Similarly, entropic contributions from other side chain groups were not considered in the analysis.

Figure 7. Conformational entropy of protein-protein complex formation. Calmodulin acts as a signaling protein and therefore interacts with various targets in the cell. Several structures of calmodulin-protein complexes are known. When determining the order parameter of methyl groups in the bound and unbound state, a linear behavior was found between total and solvent entropy changes and the change in the local disorder of the methyl groups. Black dots display entropy changes in calmodulin-protein complexes. The white dot corresponds to a more hydrophobic protein showing an unequal interaction type, the arrow indicates an altered value after hydrophobic cluster correction (figure reproduced with permission of reference [35]).

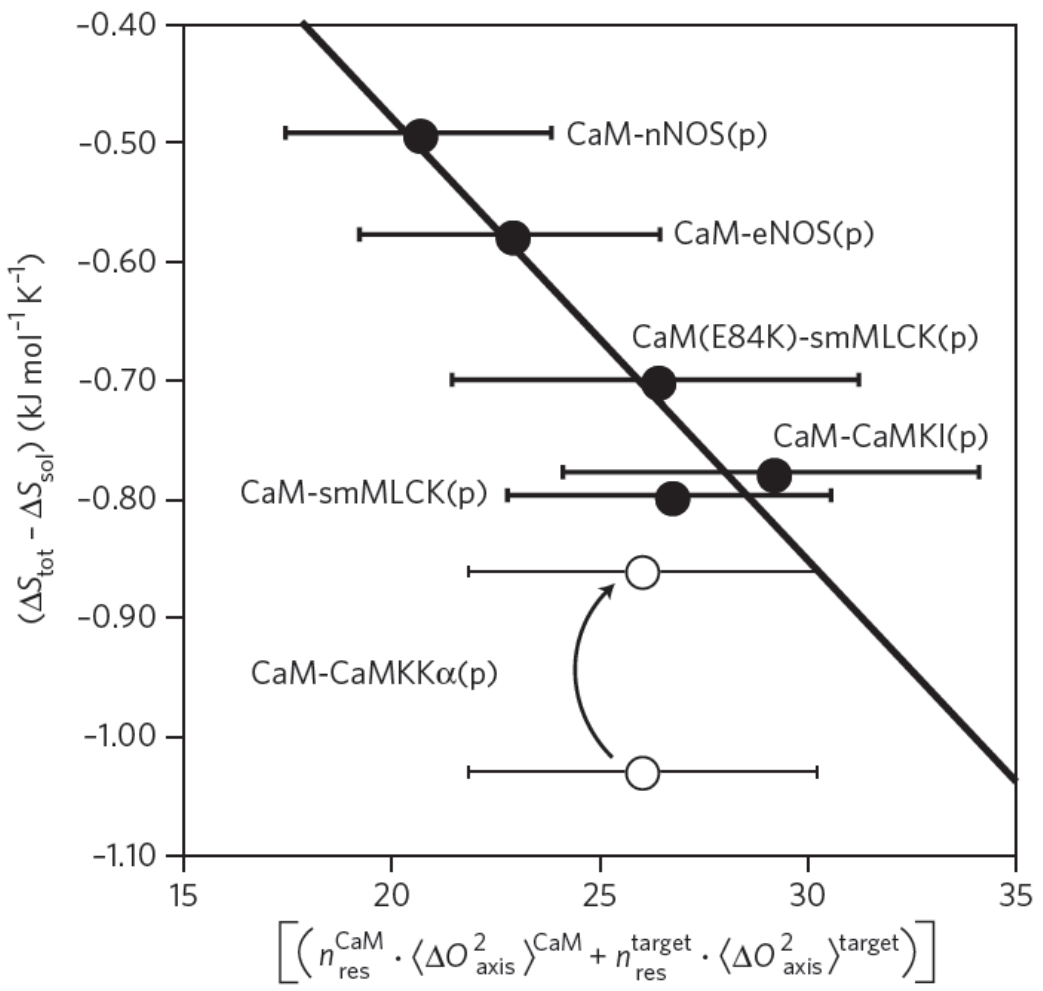


It is not clear if the insights from calmodulin binding events can be generalized, but the potential of experimentally decompose the overall entropy into its individual components is remarkable. With this method, energetic contributions to binding events can be studied in a detailed, residue specific manner.

The above over-simplified characterization of fluctuations and dynamics based on their different time scales that can be studied by NMR in reality often are not very clear-cut. There are situations where contributions from dynamics at different time scales overlap. Typically, in these cases only the upper or lower limits of the motions can be defined. Interestingly, there are other NMR parameters that envelope the full range of time scales, from nanoseconds all the way to seconds.

\section{NMR Parameters that Are Sensitive to a Broad Range of Time Scales}

In fact all of NMR parameters are ensemble and time averaged quantities. They are sensitive to dynamic processes with the slowest rate equaling the inverse of the magnitude of the nuclear interaction that is being probed. Paramagnetic relaxation enhancement and residual dipolar coupling are good examples of parameters that have been used to describe ensemble representation of protein conformations in solution by NMR.

Paramagnetic relaxation enhancement (PRE) measurement requires introduction of moieties with unpaired electrons, such as stable nitroxyl radicals or transition metal compounds, attached in most cases via reactive cysteine thiol groups. Due to the dipolar interaction between the nuclear and electron spins, an increased nuclear relaxation rate will be observed for nuclei in spatial proximity up to $30 \AA$ away from the paramagnetic center. The change in the observed nuclear relaxation time of a certain residue displays an average over all populated conformations. Since this PRE effects can be measured at a large number of sites in the protein, the ensemble conformations often times can be determined assuming the ensemble size is low enough. This is unique to solution NMR and this type of information is not accessible by other methods.

Clore and coworkers demonstrated the ensemble-PRE method by investigating protein and DNA interactions (Figure 8). Two proteins with similar fold and function as transcription factors were studied. The protein SRY (sex-determinating-region Y) binds to the minor groove of a specific double-stranded DNA sequence. The latter was modified to incorporate spin labels. This allowed the measurement of residue specific PREs on the protein. The result showed a clear distance dependent single binding site of the DNA on the protein [37]. This data confirmed the high specificity of the interaction resulting in a single conformationally distinct binding region and provided the control reference on a system without the existence of an ensemble.

A similar protein, HMGB-1A, displayed contrasting PRE rates when interacting with its spin labeled target DNA. The result showed rather uniformly distributed enhanced relaxation rates throughout the protein. Analysis of the data revealed weak binding to all possible sites of the DNA duplex. The method collected different distance dependent states of the protein in a single measurement. The measured PREs of the protein can be fit to different possible positions of the protein on the DNA and represents the average ensemble distribution in solution. The HMGB-1A was found to slide along the nucleotides with low specificity rather than binding to a distinct region [38]. 
Figure 8. PRE ensemble approach using spin labeled DNA. To study the binding of the two proteins HMGB-1A (A) and SRY (B) spin labeled DNA was used. On the left, residue specific PREs from site specific spin labels are plotted. Relaxation rates from two opposing modifications in the DNA are printed in red and in blue. In the non-specific HMGB-1A/DNA complex diagram, green asterisks on top indicate too strong line broadening and on the bottom and in black PREs from a diamagnetic control are shown. HMGB-1A displays a residue specific PRE profile that can only be fitted to multiple binding sites since the data cannot be explained by any single, specific protein-DNA complex. In contrast, SRY shows a clear singular binding site and the residue specific PRE data was used to refine the solution structure of the protein-DNA complex. On the right, a schematic illustration of the binding behavior is shown. (Figure reproduced with permission of reference [39].)

A

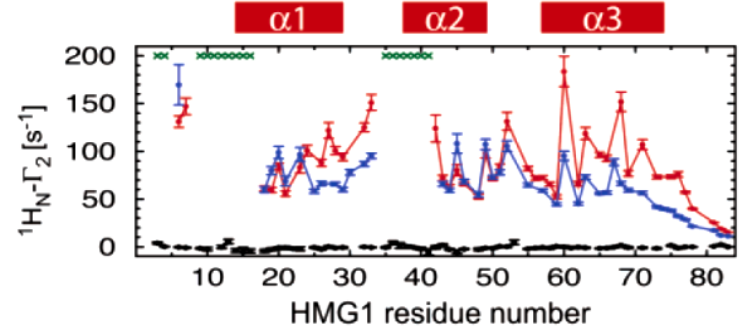

B SRY

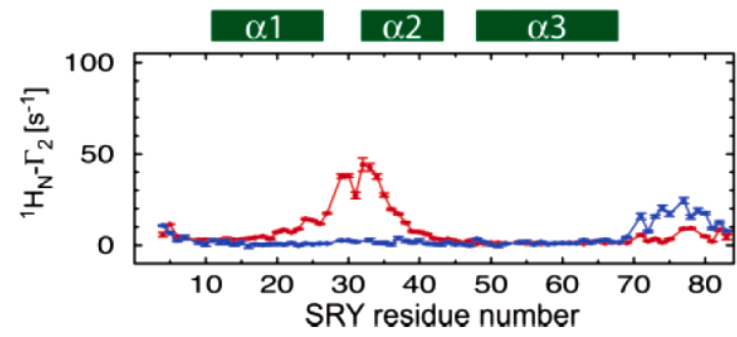

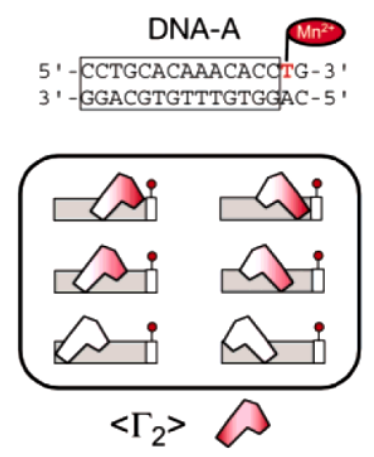



DNA-B

5' - CACCTGCACAAACACC- 3 ' 3' - GTGGACGTGTTTGTGG- 5 '
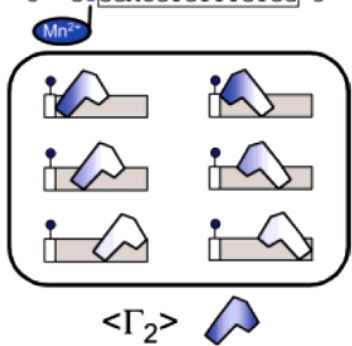

$<\Gamma_{2}>\bigotimes$



The PRE approach was found to be useful also for transient protein-protein interactions [40], transient large-scale domain reorientations [41] or ultra weak interactions [42] that so far escaped their detection due to their short lifetime.

Another approach for a dynamic ensemble description uses residual dipolar couplings (RDCs) as an experimental probe. RDCs have been found to be sensitive to motion up to milliseconds [43]. Dipolar coupling depends on the distance between the interacting nuclear dipoles and the orientation of the interaction vector with respect to the magnetic field. This coupling is averaged to zero when the protein is tumbling isotropically in solution. An anisotropic medium can be introduced into the sample and has been shown to create a small alignment of the protein in the magnetic field and therefore a residual non-zero dipolar coupling. RDCs contain long range orientational information [44].

Lange and Lakomek et al. have calculated an ensemble of NMR structures of ubiquitin making extensive use of experimental RDCs by translating motional averaged parameters into structural data [45]. Ensembles were calculated that fulfilled both short distance NOE and RDC data of a protein. 
The resulting high-resolution ensemble displayed structural heterogeneity at various parts of the protein. Analogous to the Lipari-Szabo order parameter [32,33] for very fast motion, a slow motion order parameter $\left(\mathrm{S}^{2}\right.$ supra $)$ was defined according to structural variation of the derived ensemble (Figure 9).

Figure 9. Structural ensemble of ubiquitin displaying $\mu$ s-dynamics. A dynamic structural ensemble of ubiquitin derived from RDC measurements is shown. The color coding demonstrates structural heterogeneity, ranging from dark blue and no dynamics to dark red and high dynamics. The unstructured terminus displays high flexibility but highly dynamic residues can also be found for structured regions of the protein. Some loop regions show high dynamics and sample several conformations within the microseconds range. Similar structural heterogeneity can also be found for different ubiquitin X-ray structures when complexed to interaction partners. Principal component analysis revealed that a collective motion predominantly in the interface region of complexes is present in solution. Several lysine residues known to be involved in polyubiquitination processes show significant dynamics [46] (figure reproduced with permission of reference [45]).

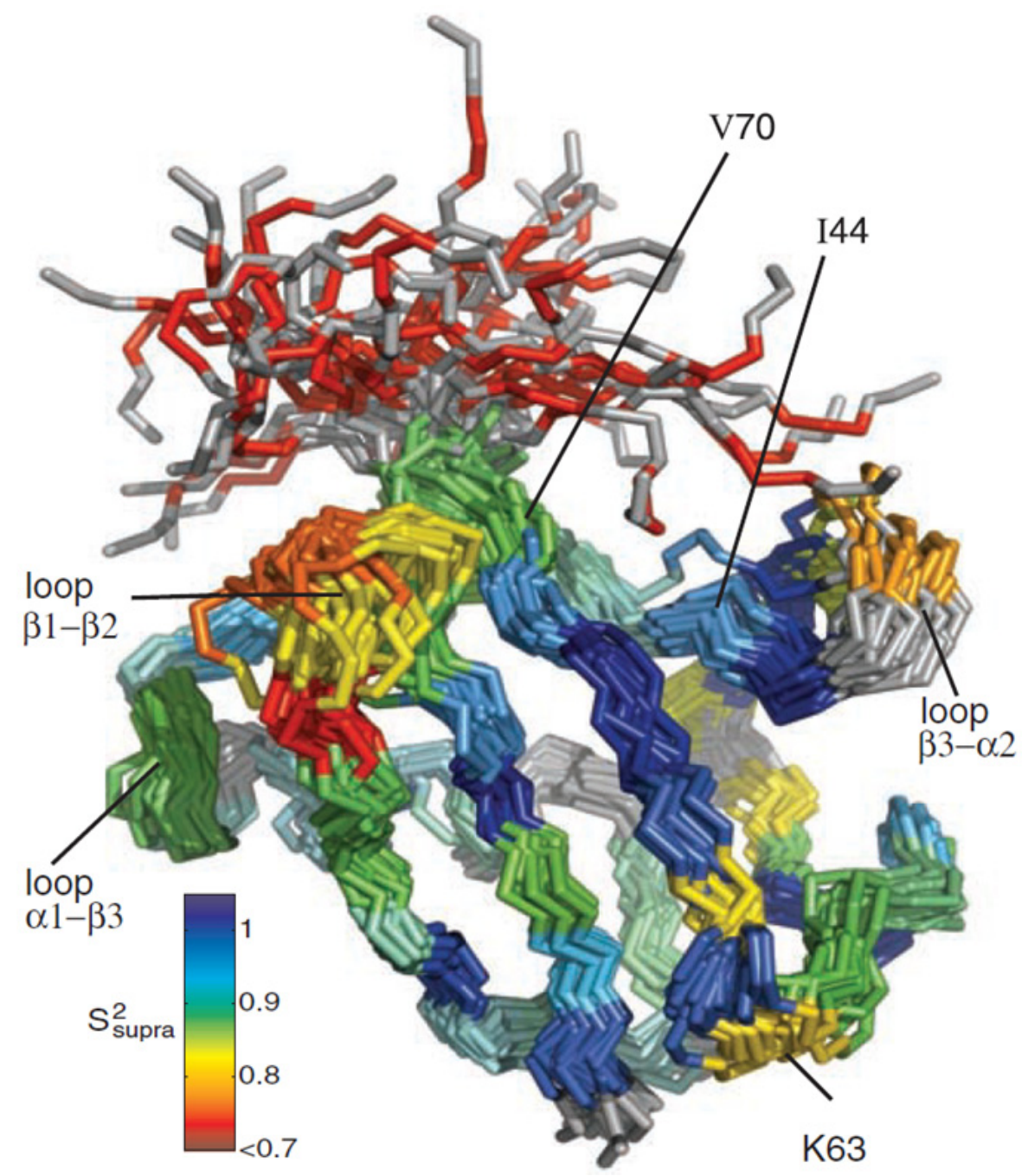


They further showed that structural heterogeneity observed in the ensemble correlated well with the conformations found in various X-ray structures of ubiquitin complexes. Regions of high flexibility show tendency for intermolecular interactions whereas the rigid helix of ubiquitin is almost entirely excluded from binding events. This observation indicates that interface contact formation is strongly biased by structural fluctuations in solution. Therefore, the results of this study suggest that the different conformations of ubiquitin found in its complexes, are already populated in solution.

Although in many cases it is quite difficult to translate dynamic parameters to a structural depiction, various NMR methods currently available can provide an ensemble representation of the system. The development of new methods is continuing and will allow further characterization in improved details of protein dynamics in solution.

\section{Conclusions}

With these recent advances in NMR we can now probe a broad range of dynamic processes in molecules of an increasing molecular size. In fact recent studies of a $670 \mathrm{kDa}$ archaeal proteasome identified dynamic contributions of the flexible termini as regulatory control whose equilibrium is carefully modulated by its interaction partners [7]. Dynamic rearrangements between open and blocked states form the basis of a peptide degrading process as revealed by analysis of intrinsic dynamics covering a broad range of timescales in combination with the PRE-ensemble approach [47]. In general, the discussed methods are not restricted to proteins and are also successfully applied to other biomolecules such as nucleotides [48]. Several previously proposed models of molecular recognition have been improved by careful analysis of dynamic contributions. The static key-lock model was first revised to the induced fit model where the receptor was thought to adjust its conformation by binding of the substrate. Although to some extend this might be the case [49,50], recent NMR investigations showed the existence of sub-states even in the absence of interaction partners.

NMR dynamic parameters will allow precise thermodynamic as well as kinetic parameters to be determined. Recent studies suggest that the contribution of residue specific conformational entropic fluctuations to binding events seemed to be underestimated in the past [51]. The possibility of determining different entropic contributions between interaction partners in a residue specific manner from general free binding energies provides an entirely different viewpoint of the binding processes. This new perspective allows a different and improved approach to rational drug design by knowing the detailed binding energies of a substrate. More importantly, the information provided by these new NMR approaches can form a foundation for a more thorough theoretical or modeling development of a number of long and outstanding fundamental questions related to allostery, signal transduction, and even protein folding which are important to provide a more comprehensive perspective on cellular functions.

\section{Acknowledgements}

C. G. thanks the Austrian Science Fund (FWF) through grant W901-B05 DK: Molecular Enzymology and is a grateful recipient of a DOC-fellowship of the Austrian Academy of Sciences at the Institute of Chemistry at the Karl-Franzens-University of Graz. This work was supported by the Intramural Research Program of National Heart, Lung, and Blood Institute of NIH to N. T. 


\section{References}

1. Amzel, L.M.; Huang, C.-H.; Mandelker, D.; Lengauer, C.; Gabelli, S.B.; Vogelstein, B. Structural comparisons of class I phosphoinositide 3-kinases. Nat. Rev. Cancer 2008, 8, 665-669.

2. Gabelli, S.; Huang, C.-H.; Mandelker, D.; Schmidt-Kittler, O.; Vogelstein, B.; Amzel, L.M. Structural effects of oncogenic PI3Ka mutations. Curr. Top. Microbiol. Immunol. 2010, 347, 43-53.

3. Koshland, D.E. Application of a theory of enzyme specificity to protein synthesis. Proc. Natl. Acad. Sci. U. S. A. 1958, 44, 98-104.

4. Henzler-Wildman, K.; Kern, D. Dynamic personalities of proteins. Nature 2007, 450, 964-972.

5. Schotte, F.; Soman, J.; Olson, J.S.; Wulff, M.; Anfinrud, P.A. Picosecond time-resolved X-ray crystallography: Probing protein function in real time. J. Struct. Biol. 2004, 147, 235-246.

6. Cammarata, M.; Levantino, M.; Schotte, F.; Anfinrud, P.A.; Ewald, F.; Choi, J.; Cupane, A.; Wulff, M.; Ihee, H. Tracking the structural dynamics of proteins in solution using time-resolved wide-angle X-ray scattering. Nat. Meth. 2008, 5, 881-886.

7. Sprangers, R.; Kay, L.E. Quantitative dynamics and binding studies of the $20 \mathrm{~S}$ proteasome by NMR. Nature 2007, 445, 618-622.

8. Sakakibara, D.; Sasaki, A.; Ikeya, T.; Hamatsu, J.; Hanashima, T.; Mishima, M.; Yoshimasu, M.; Hayashi, N.; Mikawa, T.; Walchli, M.; et al. Protein structure determination in living cells by in-cell NMR spectroscopy. Nature 2009, 458, 102-105.

9. Selenko, P.; Serber, Z.; Gadea, B.; Ruderman, J.; Wagner, G. Quantitative NMR analysis of the protein G B1 domain in Xenopus laevis egg extracts and intact oocytes. Proc. Natl. Acad. Sci. U. S. A. 2006, 103, 11904-11909.

10. Dmitri I Svergun, M.H.J.K. Small-angle scattering studies of biological macromolecules in solution. Rep. Progr. Phys. 2003, 66, 1735-1782.

11. Putnam, C.D.; Hammel, M.; Hura, G.L.; Tainer, J.A. X-ray solution scattering (SAXS) combined with crystallography and computation: Defining accurate macromolecular structures, conformations and assemblies in solution. Q. Rev. Biophys. 2007, 40, 191-285.

12. Neuman, K.C.; Nagy, A. Single-molecule force spectroscopy: Optical tweezers, magnetic tweezers and atomic force microscopy. Nat. Meth. 2008, 5, 491-505.

13. Lubitz, W.; Reijerse, E.; van Gastel, M. [NiFe] and [FeFe] hydrogenases studied by advanced magnetic resonance techniques. Chem. Rev. 2007, 107, 4331-4365.

14. Perozo, E.; Marien, D.; Cortes; Cuello, L.G. Structural rearrangements underlying K+-channel activation gating. Science 1999, 285, 73-78.

15. Englander, S.W.; Kallenbach, N.R. Hydrogen exchange and structural dynamics of proteins and nucleic acids. Q. Rev. Biophys. 1983, 16, 521-655.

16. Bai, Y.; Sosnick, T.; Mayne, L.; Englander, S. Protein folding intermediates: Native-state hydrogen exchange. Science 1995, 269, 192-197.

17. Krishna, M.M.G.; Lin, Y.; Rumbley, J.N.; Walter Englander, S. Cooperative omega loops in cytochrome c: Role in folding and function. J. Mol. Biol. 2003, 331, 29-36.

18. Krishna, M.M.G.; Hoang, L.; Lin, Y.; Englander, S.W. Hydrogen exchange methods to study protein folding. Methods 2004, 34, 51-64. 
19. Schanda, P.; Brutscher, B. Very fast two-dimensional NMR spectroscopy for real-time investigation of dynamic events in proteins on the time scale of seconds. J. Am. Chem. Soc. 2005, $127,8014-8015$.

20. Lu, K.P.; Finn, G.; Lee, T.H.; Nicholson, L.K. Prolyl cis-trans isomerization as a molecular timer. Nat. Chem. Biol. 2007, 3, 619-629.

21. Lummis, S.C.R.; Beene, D.L.; Lee, L.W.; Lester, H.A.; Broadhurst, R.W.; Dougherty, D.A. Cis-trans isomerization at a proline opens the pore of a neurotransmitter-gated ion channel. Nature 2005, 438, 248-252.

22. Loria, J.P.; Rance, M.; Palmer, A.G. A A relaxation-compensated Carr-Purcell-Meiboom-Gill sequence for characterizing chemical exchange by NMR spectroscopy. J. Am. Chem. Soc. 1999, 121, 2331-2332.

23. Palmer Iii, A.G.; Kroenke, C.D.; Loria, J.P. [10] Nuclear magnetic resonance methods for quantifying microsecond-to-millisecond motions in biological macromolecules. In Methods in Enzymology; James, T.L., Dötsch, V., Schmitz, U., Eds.; Academic Press: San Diego, CA, USA, 2001; Volume 339, pp. 204-238.

24. Volkman, B.F.; Lipson, D.; Wemmer, D.E.; Kern, D. Two-state allosteric behavior in a singledomain signaling protein. Science 2001, 291, 2429-2433.

25. Lei, M.; Velos, J.; Gardino, A.; Kivenson, A.; Karplus, M.; Kern, D. Segmented transition pathway of the signaling protein nitrogen regulatory protein C. J. Mol. Biol. 2009, 392, 823-836.

26. Gardino, A.K.; Villali, J.; Kivenson, A.; Lei, M.; Liu, C.F.; Steindel, P.; Eisenmesser, E.Z.; Labeikovsky, W.; Wolf-Watz, M.; Clarkson, M.W.; et al. Transient non-native hydrogen bonds promote activation of a signaling protein. Cell 2009, 139, 1109-1118.

27. Bhabha, G.; Lee, J.; Ekiert, D.C.; Gam, J.; Wilson, I.A.; Dyson, H.J.; Benkovic, S.J.; Wright, P.E. A dynamic knockout reveals that conformational fluctuations influence the chemical step of enzyme catalysis. Science 2011, 332, 234-238.

28. Boehr, D.D.; McElheny, D.; Dyson, H.J.; Wright, P.E. The dynamic energy landscape of dihydrofolate reductase catalysis. Science 2006, 313, 1638-1642.

29. Mulder, F.A.A.; Mittermaier, A.; Hon, B.; Dahlquist, F.W.; Kay, L.E. Studying excited states of proteins by NMR spectroscopy. Nat. Struct. Mol. Biol. 2001, 8, 932-935.

30. Bouvignies, G.; Vallurupalli, P.; Hansen, D.F.; Correia, B.E.; Lange, O.; Bah, A.; Vernon, R.M.; Dahlquist, F.W.; Baker, D.; Kay, L.E. Solution structure of a minor and transiently formed state of a T4 lysozyme mutant. Nature 2011, 477, 111-114.

31. Henzler-Wildman, K.A.; Thai, V.; Lei, M.; Ott, M.; Wolf-Watz, M.; Fenn, T.; Pozharski, E.; Wilson, M.A.; Petsko, G.A.; Karplus, M.; et al. Intrinsic motions along an enzymatic reaction trajectory. Nature 2007, 450, 838-844.

32. Lipari, G.; Szabo, A. Model-free approach to the interpretation of nuclear magnetic resonance relaxation in macromolecules. 1. Theory and range of validity. J. Am. Chem. Soc. 1982, 104, 4546-4559.

33. Lipari, G.; Szabo, A. Model-free approach to the interpretation of nuclear magnetic resonance relaxation in macromolecules. 2. Analysis of experimental results. J. Am. Chem. Soc. 1982, 104, 4559-4570. 
34. Stone, M.J. NMR relaxation studies of the role of conformational entropy in protein stability and ligand binding. Accounts Chem. Res. 2001, 34, 379-388.

35. Marlow, M.S.; Dogan, J.; Frederick, K.K.; Valentine, K.G.; Wand, A.J. The role of conformational entropy in molecular recognition by calmodulin. Nat. Chem. Biol. 2010, 6, $352-358$.

36. Lee, A.L.; Kinnear, S.A.; Wand, A.J. Redistribution and loss of side chain entropy upon formation of a calmodulin-peptide complex. Nat. Struct. Mol. Biol. 2000, 7, 72-77.

37. Iwahara, J.; Schwieters, C.D.; Clore, G.M. Ensemble approach for NMR structure refinement against $1 \mathrm{H}$ paramagnetic relaxation enhancement data arising from a flexible paramagnetic group attached to a macromolecule. J. Am. Chem. Soc. 2004, 126, 5879-5896.

38. Iwahara, J.; Clore, G.M. Detecting transient intermediates in macromolecular binding by paramagnetic NMR. Nature 2006, 440, 1227-1230.

39. Iwahara, J.; Schwieters, C.D.; Clore, G.M. Characterization of nonspecific protein-DNA interactions by $1 \mathrm{H}$ paramagnetic relaxation enhancement. J. Am. Chem. Soc. 2004, 126, $12800-12808$.

40. Tang, C.; Iwahara, J.; Clore, G.M. Visualization of transient encounter complexes in proteinprotein association. Nature 2006, 444, 383-386.

41. Tang, C.; Schwieters, C.D.; Clore, G.M. Open-to-closed transition in apo maltose-binding protein observed by paramagnetic NMR. Nature 2007, 449, 1078-1082.

42. Tang, C.; Ghirlando, R.; Clore, G.M. Visualization of transient ultra-weak protein self-association in solution using paramagnetic relaxation enhancement. J. Am. Chem. Soc. 2008, 130, 4048-4056.

43. Tolman, J.R.; Flanagan, J.M.; Kennedy, M.A.; Prestegard, J.H. NMR evidence for slow collective motions in cyanometmyoglobin. Nat. Struct. Biol. 1997, 4, 292-297.

44. Tjandra, N.; Bax, A. Direct measurement of distances and angles in biomolecules by NMR in a dilute liquid crystalline medium. Science 1997, 278, 1111-1114.

45. Lange, O.F.; Lakomek, N.-A.; Farès, C.; Schröder, G.F.; Walter, K.F.A.; Becker, S.; Meiler, J.; Grubmüller, H.; Griesinger, C.; de Groot, B.L. Recognition dynamics up to microseconds revealed from an RDC-derived ubiquitin ensemble in solution. Science 2008, 320, 1471-1475.

46. Lakomek, N.A.; Lange, O.F.; Walter, K.F.; Farès, C.; Egger, D.; Lunkenheimer, P.; Meiler, J.; Grubmüller, H.; Becker, S.; de Groot, B.L.; et al. Residual dipolar couplings as a tool to study molecular recognition of ubiquitin. Biochem. Soc. Trans. 2008, 36, 1433-1437.

47. Religa, T.L.; Sprangers, R.; Kay, L.E. Dynamic regulation of archaeal proteasome gate opening as studied by TROSY NMR. Science 2010, 328, 98-102.

48. Bothe, J.R.; Nikolova, E.N.; Eichhorn, C.D.; Chugh, J.; Hansen, A.L.; Al-Hashimi, H.M. Characterizing RNA dynamics at atomic resolution using solution-state NMR spectroscopy. Nat. Meth. 2011, 8, 919-931.

49. Wlodarski, T.; Zagrovic, B. Conformational selection and induced fit mechanism underlie specificity in noncovalent interactions with ubiquitin. Proc. Natl. Acad. Sci. U. S. A. 2009, 106, 19346-19351.

50. Pistolesi, S.; Tjandra, N. Temperature dependence of molecular interactions involved in defining stability of glutamine binding protein and its complex with 1-Glutamine. Biochemistry 2011, 51, $643-652$. 
51. Frederick, K.K.; Marlow, M.S.; Valentine, K.G.; Wand, A.J. Conformational entropy in molecular recognition by proteins. Nature 2007, 448, 325-329.

(C) 2012 by the authors; licensee MDPI, Basel, Switzerland. This article is an open access article distributed under the terms and conditions of the Creative Commons Attribution license (http://creativecommons.org/licenses/by/3.0/). 